\title{
SEPARATION THEOREMS FOR SOME PLANE-LIKE SPACES
}

\author{
BY \\ STEVE ARMENTROUT
}

In this paper, certain separation theorems, which hold in spaces satisfying R. L. Moore's Axioms 0 and 1-5 of [2], are established for a class of spaces in which there do not necessarily exist either arcs or simple closed curves. The spaces considered satisfy axioms suggested by those of Moore.

Consider a nondegenerate complete metric space $S$ which satisfies the following axioms from [2]:

Axiom 2. $S$ is locally connected.

Axiom 3. $S$ is connected and has no cut point.

Axгом 4. If $J$ is a simple closed curve in $S$, then $S-J$ has exactly two components and $J$ is the boundary of each of them.

AxIom 5. If $x$ and $y$ are distinct points and $U$ is an open set containing $x$, then there is a simple closed curve $J$ in $U$ such that $J$ separates $x$ from $y$.

It will be shown that even with a weakening of these conditions on $S$, the following separation theorems hold: (1) If neither of the compact point sets $H$ and $K$ separates the two points $x$ and $y$, and either $H$ and $K$ are disjoint or $H \cap K$ is connected, then $H \cup K$ does not separate $x$ and $y$. (2) If the common part of the two compact continua $H$ and $K$ is not connected, then $H \cup K$ separates $S$.

Principally, the conditions above are weakened by the omission of the stipulation that $S$ be complete. It is assumed instead that $S$ is locally compactly connected (a point set $K$ in $S$ is compactly connected if and only if each two points of $K$ belong to a compact continuum in $K$ ). As an example in $\$ 4$ shows, in a locally compactly connected metric space, there does not necessarily exist an arc. However, there exist chains of compact continua, and sets which are the unions of links of such chains serve as substitutes for arcs. The sets which serve as substitutes for simple closed curves are the unions of the links of closed chains of compact continua. Moore's Axioms 4 and 5 are replaced by analogous axioms for closed chains.

This problem was suggested to the writer by R. L. Moore, and the development presented here is along lines similar to that in [2] which leads to the separation theorems mentioned above.

1. Consequences of Axioms 1 and 2.

Axıом 1. $S$ is a nondegenerate connected metric space with no cut point.

The diameter of a bounded point set $M$ and the distance between two point sets $H$ and $K$ are denoted by $\operatorname{diam} M$ and dist $(H, K)$, respectively.

Presented to the Society, August 29, 1957 under the title, $A$ study of certain plane-like spaces without the use of arcs; received by the editors April 24, 1959 and, in revised form, January 29, 1960. 
The point set $X$ is compactly connected if and only if each two points of $X$ belong to a compact continuum contained in $X$, and $X$ is locally compactly connected if and only if for each point $p$ of $X$ and each open set $U$ containing $p$, there is a compactly connected open set $V$ containing $p$ and contained in $U$.

Aхгом 2. $S$ is locally compactly connected.

CoROllaRY 1.1. $S$ is locally connected and each connected open set is compactly connected.

If $x$ and $y$ are two distinct points, then $f$ is a chain of open sets from $x$ to $y$ if and only if $f$ is a finite collection $\left\{f_{1}, f_{2}, \cdots, f_{n}\right\}$ of open sets such that (1) $x \in f_{i}$ if and only if $i=1$, (2) $y \in f_{i}$ if and only if $i=n$, and (3) if $i$ and $j$ are positive integers, $i<j \leqq n$, then $f_{i}$ and $f_{j}$ intersect if and only if $j=i+1$. It is well known [2, Chapter I, Theorem 77] that if $M$ is a connected point set, $\mathcal{u}$ is a collection of open sets covering $M$, and $x$ and $y$ are points of $M$, then there is a chain of open sets of $u$ from $x$ to $y$.

TheOREM 1.2. Suppose that $U$ is a connected open set, $B$ is its boundary, $x \in B$, there is an open set $V$ containing $x$ such that $\bar{V} \cap B$ is compact, $W$ is an open set containing $x$, and $y \in U$. Then there exist a point $z$ of $B \cap W$ and a compact continuum $K$ containing $y$ and $z$ and such that $K-\{z\} \subset U$.

Proof. Let $T$ be the component of $W \cap V$ containing $x ; U \cup T$ is a connected open set. Let $a_{1}$ be the set of all connected open sets $N$ such that (1) $N \subset U \cup T$, (2) diam $N<1$, (3) if $N$ intersects $B$, then $\bar{N} \subset T$, and (4) if $y \in N$, then $N \subset U$. There exists a chain $\left\{U_{11}, U_{12}, \cdots, U_{1 n_{1}}\right\}$ of open sets of $a_{1}$ from $y$ to $x$. Let $j_{1}$ be the least positive integer $i$ such that $U_{1 i}$ intersects $B$. Let $R_{1}$ denote $U_{1 j_{1}}$, let $y_{1}$ be a point of $R_{1} \cap U_{1\left(j_{1}-1\right)}$, and let $x_{1}$ be a point of $B \cap R_{1}$. Let $D_{1}$ be $U\left\{U_{1 i}: i<j_{1}\right\} ; D_{1}$ is a connected open set contained in $U$. Let $a_{2}$ be the set of all connected open sets $N$ such that $\bar{N} \subset R_{1}, \operatorname{diam} N<1 / 2$, and if $y_{1} \in N$, then $N \subset U$. There is a chain $\left\{U_{21}, U_{22}, \cdots, U_{2 n_{2}}\right\}$ of connected open sets of $a_{2}$ from $y_{1}$ to $x_{1}$. Let $j_{2}$ be the least positive integer $i$ such that $U_{2 i}$ intersects $B$. Let $R_{2}$ denote $U_{2 j_{2}}$, let $y_{2}$ be a point of $R_{2} \cap U_{2\left(j_{2}-1\right)}$, and let $x_{2}$ be a point of $B \cap R_{2}$. Let $D_{2}$ be $U\left\{U_{2 i}: i<j_{2}\right\}$. Let this process be continued.

There exist a sequence $D_{1}, D_{2}, D_{3}, \cdots$ and a sequence $R_{1}, R_{2}, R_{3}, \cdots$ such that $\bar{R}_{1} \subset W \cap V$ and if $n$ is a positive integer, then (1) $D_{n}$ is a connected open set contained in $U$ and intersecting $D_{n+1}$, (2) $R_{n}$ is an open set such that $R_{n}$ intersects $B$, both $D_{n+1}$ and $\bar{R}_{n+1}$ are subsets of $R_{n}$, and diam $R_{n}<1 / n$. Since $\bar{V} \cap B$ is compact, it follows that there is one and only one point $z$ such that for each positive integer $n, z \in \bar{R}_{n} \cap B$. Clearly $z \in W \cap B$. It follows with the aid of Corollary 1.1 that there exists a sequence $K_{1}, K_{2}, K_{3}, \cdots$ such that (1) $K_{1}$ is a compact continuum containing $y$, intersecting $D_{2}$, and contained in $D_{1}$, and (2) if $n$ is a positive integer, then $K_{n+1}$ is a compact continuum intersecting both $K_{n}$ and $D_{n+2}$ and contained in $D_{n+1}$. Let $K$ be $\bigcup_{n=1}^{\infty} K_{n} \cup\{z\}$; then $K$ is a compact continuum such that $z \in K, y \in K$, and $K-\{z\} \subset U$. 
CoROllaRY 1.3. If $U$ is a connected open set, $M$ is a compact continuum intersecting $U$, and $z \in U-M$, then there is a compact continuum $K$ such that $z \in K, K \subset U$, and $K$ has one and only one point in common with $M$.

The statement that $g$ is a chain means that $g$ is a finite collection $\left\{g_{1}, g_{2}, \cdots, g_{n}\right\}$ of compact continua such that (1) if $i$ and $j$ are positive integers, $i<j \leqq n$, then $g_{i}$ and $g_{j}$ intersect if and only if $j=i+1$ and if $g_{i}$ and $g_{j}$ intersect, then they have one and only one point in common, and (2) $n$ is large. The sets $g_{1}, g_{2}, \ldots$, and $g_{n}$ are the links of the chain $g$, and $g_{1}$ and $g_{n}$ are the endlinks of $g$.

LEMMA 1.4. If $x$ and $y$ are distinct points of a connected open set $U$ and $\epsilon$ is a positive number, then there is a chain $g$ such that (1) $x$ belongs to one endlink of $g$ and $y$ belongs to the other endlink of $g$, and (2) each link of $g$ is a subset of $U$ and has diameter less than $\epsilon$.

Lemma 1.4 follows easily with the aid of Corollary 1.3.

2. Consequences of Axioms 1-3. The statement that $\alpha$ is a closed chain means that $\alpha$ is a finite set $\left\{\alpha_{1}, \alpha_{2}, \cdots, \alpha_{n}\right\}$ of compact continua such that (1) if $i$ and $j$ are positive integers, $i<j \leqq n$, then $\alpha_{i}$ and $\alpha_{j}$ intersect if and only if either $j=i+1$ or $i=1$ and $j=n$, and if $g_{i}$ and $g_{j}$ intersect, then they have one and only one point in common, and (2) $n$ is large. The sets $\alpha_{1}, \alpha_{2}, \cdots$, and $\alpha_{n}$ are the links of the closed chain $\alpha$.

If $M$ is a closed proper subset of $S$, then by a complementary domain of $M$ is meant a component of $S-M$.

If $a$ is a collection of sets, then $a^{*}$ denotes the union of the sets of the collection $a$.

Axiom 3. If $\alpha$ is a closed chain, then there exist two and only two complementary domains of $\alpha^{*}$, each of which has a boundary point in each link of $\alpha$, and every other complementary domain of $\alpha^{*}$ has for its boundary a subset of some one link of $\alpha$.

If $\alpha$ is a closed chain, then by a principal complementary domain of $\alpha^{*}$ is meant a complementary domain of $\alpha^{*}$ which has a boundary point in each link of $\alpha$. If $\alpha$ is a closed chain, then $\alpha^{*}$ has two and only two principal complementary domains.

Lемма 2.1. Suppose that $\alpha$ is a closed chain $\left\{\alpha_{1}, \alpha_{2}, \cdots, \alpha_{r}\right\}, D$ is a principal complementary domain of $\alpha^{*}, n$ is a positive integer less than $r$ such that if $g=\left\{\alpha_{1}, \alpha_{2}, \cdots, \alpha_{n}\right\}$, then both $g$ and $\alpha-$ gare chains, and $\left[(\alpha-g)^{*}-\left(\alpha_{1} \cup \alpha_{n}\right)\right]$ is connected, and $h$ is a chain $\left\{h_{1}, h_{2}, \cdots, h_{m}\right\}$ such that (1) if $1 \leqq i \leqq m$ and $1 \leqq j \leqq r$, then $h_{i}$ and $\alpha_{j}$ intersect if and only if either $(i, j)=(1,1)$ or $(i, j)$ $=(m, n)$ and if $h_{i}$ and $\alpha_{j}$ intersect, then they have only one point in common, and (2) for $1 \leqq i \leqq m,\left(h_{i}-\alpha^{*}\right) \subset D$. Then there is one and only one principal complementary domain of $(g \cup h)^{*}$ which is a subset of $D$. 
Proof. Let $E$ be the principal complementary domain of $\alpha^{*}$ distinct from $D$. Since $(g \cup h)^{*}$ and $E$ are disjoint, $E$ is a subset of a component $U$ of $S-(g \cup h)^{*}$; it is easily seen that $U$ is a principal complementary domain of $(g \cup h)^{*}$. With the aid of the hypothesis, it follows that $\left[\left(\cup_{i=n+1}^{r} \alpha_{i}\right)-\left(\alpha_{1} \cup \alpha_{n}\right)\right]$ $\subset U$. Then if $I$ is the principal complementary domain of $(g \cup h)^{*}$ distinct from $U, I$ and $\alpha^{*}$ are disjoint; since some link of $h$ is a subset of $D$, it follows that $I \subset D$. Clearly $U$ is not a subset of $D$.

Lemma 2.2. Suppose that $\alpha, g, h$, and D satisfy the hypothesis of Lemma 2.1, $g^{*}-\left(\alpha_{1} \cup \alpha_{n}\right)$ is connected, and $k$ is a chain $\left\{k_{1}, k_{2}, \cdots, k_{j}\right\}$ such that $k_{1}$ intersects a link of $g$ but does not intersect an endlink of $g, k_{j}$ intersects a link of $\alpha-g$ but does not intersect an endlink of $g$, and if $1<i<j, k_{i} \subset D$. Then $h^{*}$ and $k^{*}$ intersect.

Proof. Suppose that the notation is as in the proof of Lemma 2.1, and let $f$ be the chain whose links are those of $\alpha-g$ together with $\alpha_{1}$ and $\alpha_{n}$. Let $D_{1}$ and $D_{2}$ be the principal complementary domains of $(g \cup h)^{*}$ and $(f \cup h)^{*}$, respectively, which are subsets of $D$. It follows, with the aid of results established in the proof of Lemma 2.1, that $D_{1}$ and $D_{2}$ are disjoint.

Suppose that $h^{*}$ and $k^{*}$ are disjoint. With the aid of Theorem 1.2, it follows that there exists a chain $s$, where $s=\left\{s_{1}, s_{2}, \cdots, s_{t}\right\}$, such that (1) $s_{1}$ has one and only one point $q_{1}$ in common with $g^{*}, s_{1}-\left\{q_{1}\right\}$ is a connected subset of $D$, and $s_{1}$ intersects neither $\alpha_{1}$ nor $\alpha_{n}$, (2) $s_{t}$ has one and only one point $q_{t}$ in common with $f^{*}, s_{t}-\left\{q_{t}\right\}$ is a connected subset of $D$, and $s_{t}$ intersects neither $\alpha_{1}$ nor $\alpha_{n}$, (3) if $1<i<t$, then $s_{i} \subset D$, and (4) $h^{*}$ and $s^{*}$ are disjoint. It may be shown that $s^{*}$ intersects neither $D_{1}$ nor $D_{2}$. There exist two chains $u$ and $v$ such that (1) $u$ has the same relation to $g, h$, and $D_{1}$ as $s$ has to $g, f$, and $D$, and (2) $v$ has the same relation to $f, h$, and $D_{2}$ as $s$ has to $g, f$, and $D$. There exists a closed chain $\beta$ such that (1) each of $s, u$, and $v$ is a subset of $\beta$, (2) each link of $\beta$ is either a link of one of $s, u$, and $v$, or a subset of $\alpha^{*} \cup h^{*}$, (3) $\alpha^{*}-\beta^{*}$ has at most two components, and if there are two, $\alpha_{1}$ and $\alpha_{n}$ are contained in different components of $\alpha^{*}-\beta^{*}$, and (4) $h^{*}-\beta^{*}$ has at most two components, and if there are two, one intersects $\alpha_{1}$ and the other intersects $\alpha_{n}$. If $E$ is the principal complementary domain of $\alpha^{*}$ distinct from $D$, then $E$ and $\beta^{*}$ are disjoint, and $E$ is a subset of a component $U$ of $S-\beta^{*}$. Let $I$ be a principal complementary domain of $\beta^{*}$ such that $I$ is distinct from $U$. Now $E \cup\left(\alpha^{*}-\beta^{*}\right)$ is connected and hence is contained in $U$; it follows that $I$ and $\alpha^{*}$ are disjoint. It is easily seen that $I$ and $h^{*}$ are disjoint. Since some link of $\beta$ is a subset of $D_{1}$ and some link of $\beta$ is a subset of $D_{2}$, then $I$ intersects both $D_{1}$ and $D_{2}$. But this involves a contradiction, and Lemma 2.2 is proved.

If $x$ and $y$ are distinct points and $\alpha$ is a closed chain, then $\alpha^{*}$ Separates $x$ and $y$ if and only if $x$ and $y$ belong to different principal complementary domains of $\alpha^{*}$. If $x$ is a point, $M$ is a point set, and $\alpha$ is a closed chain, then 
$\alpha^{*}$ Separates $x$ and $M$ if and only if $x$ and $M$ are contained in different principal complementary domains of $\alpha^{*}$, and a similar definition holds for two point sets.

Theorem 2.3. Suppose that $x$ and $y$ are distinct points, $U$ is an open set, $\alpha$ is a closed chain such that $\alpha^{*}$ is a subset of $U$ and separates the points $x$ and $y$, $V$ is a connected open set such that $x \in V$, and $\bar{V}$ and $\alpha^{*}$ are disjoint, and $\epsilon$ is $a$ positive number. Then there exists a closed chain $\gamma$ such that $\gamma^{*}$ is a subset of $U$ and Separates $y$ and $\bar{V}$, and each link of $\gamma$ has diameter less than $\epsilon$.

Proof. Suppose that $\alpha=\left\{\alpha_{1}, \alpha_{2}, \cdots, \alpha_{n}\right\}$. Let $D$ and $E$ be the principal complementary domains of $\alpha^{*}$ containing $x$ and $y$, respectively. Suppose that $r$ and $t$ are positive integers, $r<t<n$, such that $r,(t-r)$, and $(n-t)$ are large. Let $A$ and $B$ denote $\alpha_{r} \cup \alpha_{r+1} \cup \alpha_{r+2}$ and $\alpha_{t} \cup \alpha_{t+1} \cup \alpha_{t+2}$, respectively. With the aid of Theorem 1.2 and Axiom 3, it follows that there exists a chain $h$, where $h=\left\{h_{1}, h_{2}, \cdots, h_{m}\right\}$, such that (1) $h_{1}$ has one and only one point $a_{0}$ in common with $A$ and does not intersect any link of $\alpha$ distinct from $\alpha_{r}$, $\alpha_{r+1}$, and $\alpha_{r+2}$, (2) $h_{m}$ has one and only one point $a_{m}$ in common with $B$ and does not intersect any link of $\alpha$ distinct from $\alpha_{t}, \alpha_{t+1}$, and $\alpha_{t+2}$, (3) if, for $1 \leqq i<m, a_{i}$ is the point common to $h_{i}$ and $h_{i+1}$, then, for $1 \leqq i \leqq m, h_{i}$ is irreducible between $a_{i-1}$ and $a_{i}$, and (4) $h^{*}-\left\{a_{0}, a_{m}\right\} \subset D$. There exists a chain $k$, where $k=\left\{k_{1}, k_{2}, \cdots, k_{j}\right\}$, such that (1) $k_{1}$ has one and only one point $b_{0}$ in common with $A$ and does not intersect any link of $\alpha$ distinct from $\alpha_{r}$, $\alpha_{r+1}$, and $\alpha_{r+2}$, (2) $k_{j}$ has one and only one point $b_{m}$ in common with $B$ and does not intersect any link of $\alpha$ distinct from $\alpha_{t}, \alpha_{t+1}$, and $\alpha_{t+2}$, (3) if, for $1 \leqq i<j, b_{i}$ is the point common to $k_{i}$ and $k_{i+1}$, then for $1 \leqq i \leqq j, k_{i}$ is irreducible between $b_{i-1}$ and $b_{i}$, and (4) $k^{*}-\left\{b_{0}, b_{j}\right\} \subset E$. Since $h^{*}-\left\{a_{0}, a_{m}\right\} \subset D$, there exists a compact continuum $H$ lying in $D$ containing $x$, and intersecting $h^{*}$. Similarly, there is a compact continuum $K$ lying in $E$, containing $y$, and intersecting $k^{*}$. There exists a connected open set $R$ such that $R \subset U, \alpha_{1} \subset R$, $R \cap \alpha^{*} \subset \alpha_{2} \cup \alpha_{1} \cup \alpha_{2}$, and $R$ intersects no one of $h^{*}, k^{*}, A, B, H, K$, and $\bar{V}$. With the aid of Theorem 1.2, it follows that there exists a chain $g_{1}$, where $g_{1}=\left\{g_{11}, g_{12}, \cdots, g_{1 n_{1}}\right\}$, such that (1) each link of $g_{1}$ is a subset of $R$ and has diameter less than $\epsilon$, (2) $g_{11}$ has one and only one point $x_{1}$ in common with $\left(\alpha-\left\{\alpha_{1}\right\}\right)^{*}$ and $x_{1} \in \alpha_{2}$, (3) $g_{1 n_{1}}$ has one and only one point $x_{2}$ in common with $\left(\alpha-\left\{\alpha_{1}\right\}\right)^{*}$ and $x_{2} \in \alpha_{n}$, and (4) if $1<i<n_{1}$, then $g_{1 i}$ and $\left(\alpha-\left\{\alpha_{1}\right\}\right)^{*}$ are disjoint. Let $\gamma_{1}$ be the closed chain $\left(\alpha-\left\{\alpha_{1}\right\}\right) \cup g_{1}$. Since $\left(h^{*}-\left\{a_{0}, a_{m}\right\}\right) \cup H$ is a connected set not intersecting $\gamma_{1}^{*}$, containing $x$, and having limit points both in $A$ and in $B$, then by Axiom 3,x belongs to some principal complementary domain of $\gamma_{1}^{*}$. By a similar argument, $y$ belongs to a principal complementary domain of $\gamma_{1}^{*}$.

Suppose that $x$ and $y$ are not Separated by $\gamma_{1}^{*}$. Then let $\beta$ be the closed chain whose links are those of $h$ and those of $k$, together with $A$ and $B$, except that if $b_{0}=a_{0}$, omit $A$ as a link, and if $b_{j}=a_{m}$, omit $B$ as a link. By 
Axiom 3, there is a principal complementary domain $I$ of $\beta^{*}$ which contains each link $\alpha_{i}$ of $\alpha$ for $1 \leqq i<(r-1)$ and $(t+1)<i \leqq n$. Clearly then $g_{1}^{*} \subset I$. Since $x$ and $y$ belong to the same principal complementary domain of $\gamma_{1}^{*}$, it may be seen that there is a chain $s$, where $s=\left\{s_{1}, s_{2}, \cdots, s_{q}\right\}$, such that (1) for some positive integer $p_{1}, 1<p_{1}<m, s_{1}$ intersects $h_{p_{1}}$, (2) for some positive integer $p_{2}, 1<p_{2}<j, s_{q}$ intersects $k_{p_{2}}$, (3) if $1<i<q$, then $s_{i}$ and $\beta^{*}$ are disjoint, and (4) $s^{*}$ and $\gamma_{1}^{*}$ are disjoint. Since $s^{*}$ intersects $\alpha^{*}$, then $s^{*}$ intersects $\alpha_{1}$ and hence for $1<i<q, s_{i} \subset I$. But this contradicts Lemma 2.2, and therefore $\gamma_{1}^{*}$ Separates $x$ and $y$.

Now $\gamma_{1}^{*}$ Separates $y$ and $\bar{V}$ and is a subset of $U$, and $\gamma_{1}$ has at most $(n-1)$ links, each of which has diameter greater than or equal to $\epsilon$. This process may be repeated, using the closed chain $\gamma_{1}$ and the link $\alpha_{2}$ of $\gamma_{1}$ in place of the closed chain $\alpha$ and the link $\alpha_{1}$ of $\alpha$. There results a closed chain $\gamma_{2}$, at most $(n-2)$ links of which have diameter greater than or equal to $\epsilon$, and such that $\gamma_{2}^{*}$ is a subset of $U$ and Separates $y$ and $\bar{V}$. Let this process continue; it terminates after a finite number of steps with a closed chain $\gamma$ such that each link of $\gamma$ has diameter less than $\epsilon, \gamma^{*} \subset U$, and $\gamma^{*}$ Separates $y$ and $\bar{V}$.

Suppose that $p$ is a point and $\alpha$ is a closed chain. Then $\alpha$ is bounded with respect to $p$ if and only if $p$ belongs to some principal complementary domain of $\alpha^{*}$. If $\alpha$ is bounded with respect to $p$, then the interior, with respect to $p$, of $\alpha^{*}$ is the principal complementary domain of $\alpha^{*}$ which does not contain $p$, and the exterior, with respect to $p$, of $\alpha^{*}$ is the principal complementary domain of $\alpha^{*}$ which contains $p$.

Throughout the remainder of this section, let $\omega$ be some definite point, and let the terms "bounded," "interior," and "exterior" mean "bounded with respect to $\omega$," "interior with respect to $\omega$," and "exterior with respect to $\omega$, , respectively. If $\alpha$ is a bounded closed chain, int $\alpha^{*}$ and ext $\alpha^{*}$ denote the interior and exterior, respectively, of $\alpha^{*}$.

LemMA 2.4. Suppose that $U_{1}$ and $U_{2}$ are open sets, $\alpha_{1}$ and $\alpha_{2}$ are bounded closed chains such that $\alpha_{1}^{*} \subset \dot{U}_{1}$ and $\alpha_{2}^{*} \subset U_{2}, V_{1}$ and $V_{2}$ are connected open sets such that $\omega \in V_{1} \cap V_{2}, \bar{V}_{1} \subset \operatorname{ext} \alpha_{1}^{*}$, and $\bar{V}_{2} \subset \operatorname{ext} \alpha_{2}^{*}$, and $p \in$ (int $\left.\alpha_{1}^{*}\right) \cap$ (int $\left.\alpha_{2}^{*}\right)$. Then there exist two bounded closed chains $\gamma_{1}$ and $\gamma_{2}$ such that $\gamma_{1}^{*} \subset U_{1}, \gamma_{2}^{*} \subset U_{2}$, $\bar{V}_{1} \subset \operatorname{ext} \gamma_{1}^{*}, \bar{V}_{2} \subset \operatorname{ext} \gamma_{2}^{*}, p \in\left(\right.$ int $\left.\gamma_{1}^{*}\right) \cap\left(\right.$ int $\left.\gamma_{2}^{*}\right)$, and if $x$ is a link of $\gamma_{1}$ intersecting $\bar{V}_{2}$, then there exists a bounded closed chain $\sigma_{x}$ such that (1) each link of $\sigma_{x}$ is either a link of $\gamma_{1}$, or a link of $\gamma_{2}$ which lies, except possibly for one point, in the interior of $\gamma_{1}^{*}$, and (2) $p$ belongs to the interior of $\sigma_{x}^{*}$ and $x \subset\left(\operatorname{ext} \sigma_{x}^{*}\right)$.

Proof. Let $W_{1}$ be an open set such that $\alpha_{1}^{*} \subset W_{1}, \bar{W}_{1} \subset U_{1}$, and $\bar{W}_{1}$ and $\bar{V}_{1}$ are disjoint. With the aid of Theorem 2.3, it follows that there exists a closed chain $\beta_{1}$ such that (1) $\beta_{1}^{*} \subset W_{1}$ and $p \in \operatorname{int} \beta_{1}^{*}$, (2) each link of $\beta_{1}$ which intersects $\bar{V}_{2}$ belongs to a chain of links of $\beta_{1}$, no one of which intersects $\alpha_{2}^{*}$, and (3) each link of $\beta_{1}$ is irreducible between the two points it has in common 
with other links of $\beta_{1}$. By taking unions of links of $\beta_{1}$, if necessary, there results a closed chain $\mu_{1}$ such that $\mu_{1}^{*} \subset U_{1}, p \in$ int $\mu_{1}^{*}, \mu_{1}^{*}$ and $\bar{V}_{1}$ are disjoint, and $\alpha_{2}^{*}$ contains no point common to two links of $\mu_{1}$. Let $F$ be an open set such that $\alpha_{2}^{*} \subset F$ and $\bar{F}$ contains no point common to two links of $\mu_{1}$. As in the proof of Theorem 2.3, it may be shown that there is a closed chain $\gamma_{2}$ such that (1) $\gamma_{2}^{*} \subset F \cap U_{2}, \gamma_{2}^{*}$ and $\bar{V}_{2}$ are disjoint, and $p \in$ int $\gamma_{2}^{*}$, (2) each link of $\gamma_{2}$ is irreducible between the two points it has in common with other links of $\gamma_{2}$, (3) no link of $\gamma_{2}$ intersects two distinct links of $\mu_{1}$, and (4) the links of $\gamma_{2}$ have small diameters. Let $\gamma_{1}$ be the closed chain such that $y$ is a link of $\gamma_{1}$ if and only if for some link $z$ of $\mu_{1}, y$ is the union of $z$ and all the links of $\boldsymbol{\gamma}_{2}$ which intersect $z$.

Suppose that $x$ is a link of $\gamma_{1}$ intersecting $\bar{V}_{2}$. It may be shown, principally with the aid of Lemma 2.1 or of a modification of it, that there exist a chain $h_{x}$ of links of $\gamma_{2}$ and a closed chain $\sigma_{x}$ whose links are those of $h_{x}$ together with certain links of $\gamma_{1}$ such that $\sigma_{x}$ satisfies the conditions of the conclusion of Lemma 2.4 .

Theorem 2.5. Suppose that $U_{1}$ and $U_{2}$ are open sets, $\alpha_{1}$ and $\alpha_{2}$ are bounded closed chains such that $\alpha_{1}^{*} \subset U_{1}$ and $\alpha_{2}^{*} \subset U_{2}, V_{1}$ and $V_{2}$ are connected open sets such that $\omega \in V_{1} \cap V_{2}, \bar{V}_{1} \subset$ ext $\alpha_{1}^{*}$, and $\bar{V}_{2} \subset$ ext $\alpha_{2}^{*}$, and $p \in$ (int $\left.\alpha_{1}^{*}\right) \cap$ (int $\left.\alpha_{2}^{*}\right)$. Then there exists a bounded closed chain $\beta$ such that $\beta^{*} \subset U_{1} \cup U_{2}, p \in \operatorname{int} \beta^{*}$, and $\bar{V}_{1} \cup \bar{V}_{2} \subset \operatorname{ext} \beta^{*}$.

Proof. Let $\gamma_{1}$ and $\gamma_{2}$ be bounded closed chains satisfying the conditions of Lemma 2.4 relative to $U_{1}, U_{2}, V_{1}, V_{2}$, and $p$. Let $x_{1}, x_{2}, \cdots$, and $x_{n}$ be the links of $\gamma_{1}$ which intersect $\bar{V}_{2}$. By Lemma 2.4 , there is a bounded closed chain $\mu_{1}$, each link of which either is a link of $\gamma_{1}$ or is a link of $\gamma_{2}$ which, except possibly for one point, lies in the interior of $\gamma_{1}^{*}$, and such that $p \in$ int $\mu_{1}^{*}$ and $x_{1} \subset$ ext $\mu_{1}^{*}$; note that int $\mu_{1}^{*} \subset$ int $\gamma_{1}^{*}$. Now if for some positive integer $m, m$ $\leqq n, x_{m}$ intersects $\mu_{1}^{*}$, then $x_{m}$ is a link of $\mu_{1}$. Let $n_{2}$ be the least positive integer $i$ such that $x_{i}$ intersects $\mu_{1}^{*}$. Again by Lemma 2.4, there is a bounded closed chain $\mu_{2}$, each link of which either is a link of $\mu_{1}$ or is a link of $\gamma_{2}$ which, except possibly for one point, lies in the interior of $\mu_{1}^{*}$, and such that $p \in$ int $\mu_{2}^{*}, x_{n_{2}} \subset \operatorname{ext} \mu_{2}^{*}$, and int $\mu_{2}^{*} \subset$ int $\mu_{1}^{*}$. Neither $x_{1}$ nor $x_{2}$ is a link of $\mu_{2}$. Let this process be continued; there results a bounded closed chain $\beta$ such that $p \in$ int $\beta^{*}, \beta^{*} \subset U_{1} \cup U_{2}$, and no one of $x_{1}, x_{2}, \cdots$, and $x_{n}$ intersects $\beta^{*}$. It follows that $\bar{V}_{1} \cup \bar{V}_{2} \subset \operatorname{ext} \beta^{*}$.

TheOREM 2.6. Suppose that $U_{1}$ and $U_{2}$ are open sets, $\alpha_{1}$ and $\alpha_{2}$ are bounded closed chains such that $\alpha_{1}^{*} \subset U_{1}$ and $\alpha_{2}^{*} \subset U_{2}$, and $V_{1}$ and $V_{2}$ are intersecting connected open sets such that $\bar{V}_{1} \subset$ int $\alpha_{1}^{*}$ and $\bar{V}_{2} \subset$ int $\alpha_{2}^{*}$. Then there exists a bounded closed chain $\gamma$ such that $\gamma^{*} \subset U_{1} \cup U_{2}$ and $\bar{V}_{1} \cup \bar{V}_{2} \subset$ int $\gamma^{*}$.

Proof. Let $q$ be a point of $V_{1} \cap V_{2}$, and apply Theorem 2.5, regarding "interior" and "exterior" as "interior with respect to $q$ " and "exterior with respect to $q$," respectively. 


\section{Consequences of Axioms 1-4.}

Axiom 4. If $x$ and $y$ are distinct points and $U$ is an open set containing $x$, then there exists a closed chain $\alpha$ such that $\alpha^{*} \subset U$ and $\alpha^{*}$ Separates $x$ and $y$.

TheOREM 3.1. Suppose that $H$ and $K$ are disjoint compact point sets, $x \in H$, and $y \in K$. Then there is a closed chain $\gamma$ such that $\gamma^{*}$ Separates $x$ and $y$ and $\gamma^{*}$ does not intersect $H \cup K$.

Proof. Let $D$ be the component of $S-K$ which contains $x$. If $p \in D$, then by Axiom 4 , there is a closed chain $\alpha_{p}$ such that $\alpha_{p}^{*}$ is a subset of $D$ and Separates $p$ and $y$. There is a connected open set $V_{p}$ such that $p \in V_{p}, \bar{V}_{p} \subset D$, and $\bar{V}_{p}$ and $\alpha_{p}^{*}$ are disjoint. Let $U$ be $\left\{V_{p}: p \in D\right\}$; since $D \cap H$ is compact, there is a finite subset $\left\{p_{1}, p_{2}, \cdots, p_{n}\right\}$ of $D \cap H$ such that $\left\{V_{p_{1}}, V_{p_{2}}, \cdots, V_{p_{n}}\right\}$ covers $D \cap H$. Let $i$ be a positive integer, $i \leqq n$. Since $D$ is connected, there is a chain $\left\{U_{i 1}, U_{i 2}, \cdots, U_{i n_{i}}\right\}$ of open sets of $v$ such that $x \in U_{i 1}$ and $U_{i n_{i}}=V_{p_{i}}$. For each positive integer $j, j \leqq n_{i}$, there is a point $p_{i j}$ of $D$ such that $U_{i j}=V_{p_{i j}}$, and let $V_{i j}$ and $\alpha_{i j}$ denote $V_{p_{i j}}$ and $\alpha_{p_{i j}}$, respectively. By repeated application of Theorem 2.6 to the chains $\alpha_{i 1}, \alpha_{i 2}, \cdots$, and $\alpha_{i n_{i}}$, it follows that there exists a closed chain $\beta_{i}$ such that $\beta_{i}^{*} \subset D, \beta_{i}^{*}$ Separates $x$ and $y$, and $U_{j=1}^{n_{i}} \bar{V}_{i j}$ and $\beta_{i}^{*}$ are disjoint. For each positive integer $i, i \leqq n$, let $W_{i}$ denote $\cup_{j=1}^{n_{i}} V_{i j}$. Since for each positive integer $i, i \leqq n, x \in W_{i}$, it follows with the aid of Theorem 2.6 that there exists a closed chain $\gamma$ such that $\gamma^{*} \subset D, \gamma^{*}$ Separates $x$ and $y$, and $\bigcup_{i=1}^{n} \bar{W}_{i}$ and $\gamma^{*}$ are disjoint. Since $H \cap D$ $\subset \bigcup_{i=1}^{n} W_{i}$, then $\gamma^{*}$ and $H$ are disjoint, and since $\gamma^{*} \subset D$, then $K$ and $\gamma^{*}$ are disjoint.

THEOREM 3.2. If $x$ and $y$ are distinct points, $H$ and $K$ are disjoint compact point sets, and neither $H$ nor $K$ separates $x$ from $y$, then $H \cup K$ does not separate $x$ from $y$.

Proof. There exist compact continua $A$ and $B$, each containing both $x$ and $y$, such that $A$ and $H$ are disjoint, and $B$ and $K$ are disjoint. Suppose that $B$ intersects $H$, for otherwise the conclusion clearly holds. Suppose that $p \in B \cap H$; since $H$ and $K \cup A$ are disjoint compact sets, then by Theorem 3.1, there is a closed chain $\gamma_{p}$ such that $\gamma_{p}^{*}$ Separates $p$ and $x$, and $\gamma_{p}^{*}$ and $H \cup K$ $\cup A$ are disjoint. Let $V_{p}$ be a connected open set containing $p$ and such that $\bar{V}_{p}$ and $\gamma_{p}^{*}$ are disjoint. Now $\left\{V_{p}: p \in B \cap H\right\}$ covers the compact set $B \cap H$ and there is a finite subset $\left\{p_{1}, p_{2}, \cdots, p_{n}\right\}$ of $B \cap H$ such that $\left\{V_{p_{1}}, V_{p_{2}}, \cdots, V_{p_{n}}\right\}$ covers $B \cap H$. Let $W$ denote $U_{i=1}^{n} V_{p_{i}} . W$ has only finitely many components and it follows, with the aid of Theorem 2.6, that there is a finite set $\left\{\beta_{1}, \beta_{2}, \cdots, \beta_{m}\right\}$ of closed chains such that (1) if $U$ is a component of $W$, then for some positive integer $j, j \leqq m, \beta_{j}^{*}$ Separates $x$ and $\bar{U}$, and (2) for each positive integer $j, j \leqq m, H \cup K \cup A$ and $\beta_{j}^{*}$ are disjoint. Let $K_{1}, K_{2}, \cdots, K_{r}$ be the components of $\bigcup_{i=1}^{m} \beta_{i}^{*}$, and let $K_{0}, K_{r+1}$, and $K_{r+2}$ denote $\{x\},\{y\}$, and $H$, respectively. Let $\delta$ be the minimum of $\left\{\operatorname{dist}\left(K_{i}, K_{j}\right): 0 \leqq i \leqq r+2,0 \leqq j \leqq r+2\right.$, and $\left.i \neq j\right\}$. 
There exists a chain $f$, where $f=\left\{f_{1}, f_{2}, \cdots, f_{d}\right\}$, such that (1) $x \in f_{1}$ and $y \in f_{d}$, (2) each link of $f$ has diameter less than $\delta / N$, where $N$ is some large positive integer, and (3) $K$ and $f^{*}$ are disjoint and $f^{*} \cap H$ is a subset of $W$. Let $s_{1}$ be the least positive integer $i$ such that $f_{i}$ intersects $U_{1=q}^{r} K_{q}$. Since no link of $f$ intersects distinct components of $\mathrm{U}_{q=1}^{r} K_{q}$, there is a positive integer $m_{1}$ such that $f_{s_{1}}$ intersects $K_{m_{1}}$ and no other component of $\mathrm{U}_{q=1}^{r} K_{q}$. Let $t_{1}$ be the largest positive integer $i$ such that $f_{i}$ intersects $K_{m_{1}}$. Note that $1<s_{1} \leqq t_{1}<d$. Let $g_{1}$ denote $\left\{f_{1}, f_{2}, \cdots, f_{s_{1}}\right\}$. Let $s_{2}$ be the least positive integer $i$ such that $i>t_{1}$ and $f_{i}$ intersects $\mathrm{U}_{q=1}^{r} K_{q}$. There is a positive integer $m_{2}$ such that $f_{s_{2}}$ intersects $K_{m_{2}}$. Let $t_{2}$ be the largest positive integer $i$ such that $f_{i}$ intersects $K_{m_{2}}$. Then $t_{1}<s_{2} \leqq t_{2}<d$. Let $g_{2}$ denote $\left\{f_{t_{1}}, f_{t_{1}+1}, \cdots, f_{s_{2}}\right\}$. Let this process be continued and suppose that $u$ is the largest positive integer $i$ such that $f_{i}$ intersects $\mathrm{U}_{q=1}^{r} K_{q}$, and let $g_{u+1}$ denote $\left\{f_{t_{u}}, f_{t_{u+1}}, \cdots, f_{d}\right\}$. It is clear that

$$
\left(\bigcup_{i=1}^{u+1} g_{i}^{*}\right) \cup\left(\bigcup_{i=1}^{u} K_{m_{i}}\right)
$$

is a continuum containing $x$ and $y$ and intersecting neither $H$ nor $K$. Therefore $H \cup K$ does not separate $x$ and $y$.

THEOREM 3.3. If $x$ and $y$ are distinct points, neither of the compact point sets $H$ and $K$ separates $x$ and $y$, and $H \cap K$ is connected, then $H \cup K$ does not separate $x$ and $y$.

Proof. There exist compact continua $A$ and $B$, each containing both $x$ and $y$, such that $A$ and $H$ are disjoint, and $K$ and $B$ are disjoint. Let $b$ be a point of $H \cap K$; by Theorem 3.1, there is a closed chain $\alpha$ such that $\alpha^{*}$ Separates $x$ and $b$ and $\alpha^{*}$ does not intersect $A \cup B \cup(H \cap K)$. There exists a connected open set $V$ such that $b \in V, H \cap K \subset V$, and $\bar{V}$ and $\alpha^{*}$ are disjoint. There is, by Theorem 2.3, a closed chain $\beta$ such that $\beta^{*}$ Separates $A \cup B$ and $\bar{V}$, and each link of $\beta$ has diameter less than $(1 / N)$ dist $(H-V, K-V)$, where $N$ is a large positive integer. There exists a finite set $\left\{f_{1}, f_{2}, \cdots, f_{j}\right\}$ such that (1) for $1 \leqq i \leqq j, f_{i}$ is a chain of links of $\beta$ and $f_{i}^{*}$ intersects at most one of $H$ and $K,(2)$ if $1 \leqq i<r \leqq j$, then $f_{i}^{*}$ and $f_{r}^{*}$ are disjoint, and (3) if a link of $\beta$ intersects $H \cup K$, then it belongs to some one of $f_{1}, f_{2}, \cdots$, and $f_{j}$. If $p \in H \cap B$, there is a closed chain $\gamma_{p}$ such that $\gamma_{p}^{*}$ Separates $p$ and $x, \gamma_{p}^{*}$ does not intersect either $A, K$, or $\bigcup_{i=1}^{j} f_{i}^{*}$, and the links of $\gamma_{p}$ have small diameters. For each point $p$ of $H \cap B$, let $U_{p}$ be a connected open set containing $p$ such that $\bar{U}_{p}$ intersects neither $\gamma_{p}^{*}$ nor $\beta^{*}$. Then there is a finite subset $\left\{p_{1}, p_{2}, \cdots, p_{n}\right\}$ of $H \cap B$ such that $\left\{U_{p_{1}}, U_{p_{2}}, \cdots, U_{p_{n}}\right\}$ covers $H \cap B$. For each positive integer $i, i \leqq n$, let $\gamma_{i}$ be $\gamma_{p_{i}}$ and let $U_{i}$ be $U_{p_{i}}$. Then it may be shown, by a process similar to that used to establish Lemma 2.4, that for each positive integer $i, i \leqq n$, there is a chain $h_{i}$ of links of $\gamma_{i}$, where $h_{i}=\left\{h_{i 1}, h_{22}, \cdots, h_{i n_{i}}\right\}$, and 
a chain $k_{i}$ of links of $\beta$, where $k_{i}=\left\{k_{i 1}, k_{i 2}, \cdots, k_{i m_{i}}\right\}$, such that if $\mu_{i}$ is the chain whose links are those of $h_{i}$ except for its endlinks, those of $k_{i}$ except for its endlinks, $h_{i 1} \cup k_{i 1}$, and $h_{i n_{i}} \cup k_{i m_{i}}$, then $\mu_{i}^{*}$ intersects neither $A$ nor $K$, and $\mu_{i}^{*}$ Separates $x$ and $\bar{U}_{\imath}$.

Since $B$ is a continuum, there is a chain $g$, one of whose endlinks contains $x$ and the other of which contains $y$, such that (1) $g^{*}$ intersects neither $K$ nor $\beta^{*}$, (2) if a link of $g$ intersects $H$, then it is a subset of $\bigcup_{i=1}^{n} U_{i}$, and (3) the links of $g$ are small. By a process similar to that used in the proof of Theorem 3.2 , it may be shown that there exist a finite set $\left\{s_{1}, s_{2}, \cdots, s_{r}\right\}$, each element of which is a chain of links of $g$, and a finite set $\left\{t_{1}, t_{2}, \cdots, t_{q}\right\}$, each element of which is a chain of links of some one of $\mu_{1}, \mu_{2}, \cdots$, and $\mu_{n}$, such that

$$
\left(\bigcup_{i=1}^{r} s_{i}^{*}\right) \cup\left(\bigcup_{i=1}^{q} t_{i}^{*}\right)
$$

is a continuum containing both $x$ and $y$ and intersecting neither $H$ nor $K$. Then $H \cup K$ does not separate $x$ and $y$.

THEOREM 3.4. If $H$ and $K$ are compact continua and $H \cap K$ is not connected, then $H \cup K$ separates $S$.

Proof. Suppose that $A$ and $B$ are closed disjoint point sets such that $H \cap K=A \cup B$. Let $x$ and $y$ be points of $A$ and $B$, respectively. By Theorems 3.1 and 2.3, there exists a closed chain $\gamma$ such that (1) $\gamma^{*}$ Separates $x$ and $y$, (2) $\gamma^{*}$ does not intersect $H \cap K$, and (3) there exists a finite set $\left\{g_{1}, g_{2}, \cdots, g_{n}\right\}$ of chains of links of $\gamma$ such that (i) $g_{1}^{*}, g_{2}^{*}, \cdots$, and $g_{n}^{*}$ are mutually disjoint, (ii) each link of $\gamma$ which in tersects $H \cup K$ belongs to some chain of $g_{1}, g_{2}, \ldots$, and $g_{n}$, and (iii) no one of $g_{1}^{*}, g_{2}^{*}, \cdots$, and $g_{n}^{*}$ intersects both $H$ and $K$, and each of them intersects one of $H$ and $K$. Let $\left\{\gamma_{n_{1}}, \gamma_{n_{2}}, \cdots, \gamma_{n_{j}}\right\}$ be the set of links of $\gamma$ which belong to no one of $g_{1}, g_{2}, \cdots$, and $g_{n}$.

Suppose that $H \cup K$ separates no two of $\gamma_{n_{1}}, \gamma_{n_{2}}, \cdots$, and $\boldsymbol{\gamma}_{n_{j}}$. Let $H_{0}$ be the union of those sets of $g_{1}^{*}, g_{2}^{*}, \cdots$, and $g_{n}^{*}$ which intersect $H$, and let $K_{0}$ be the union of the other sets of $g_{1}^{*}, g_{2}^{*}, \cdots$, and $g_{n}^{*}$. For each pair $s$ and $t$ of positive integers, $s \leqq j$ and $t \leqq j$, let $L_{s t}$ be a compact continuum intersecting both $\gamma_{n_{z}}$ and $\gamma_{n_{t}}$ and not intersecting $H \cup K$. Let $L$ be

$$
\left(\bigcup_{i=1}^{j} \gamma_{n_{i}}\right) \cup\left(\bigcup\left\{L_{s t}: 1 \leqq s \leqq j, 1 \leqq t \leqq j\right\}\right) .
$$

Clearly $L, H_{0} \cup L$, and $K_{0} \cup L$ are compact continua, $H_{0} \cup L$ and $K$ are disjoint, and $K_{0} \cup L$ and $H$ are disjoint. Therefore neither $H_{0} \cup L$ nor $K_{0} \cup L$ separates $x$ from $y$. However $\left(H_{0} \cup L\right) \cup\left(K_{0} \cup L\right)$ has $\gamma^{*}$ as a subset and contains neither $x$ nor $y$, and hence separates $x$ and $y$. Since the common part of $H_{0} \cup L$ and $K_{0} \cup L$ is the continuum $L$, this contradicts Theorem 3.3. It follows that $H \cup K$ separates $S$. 
4. An example. In this section, there is described an example of a space satisfying Axioms 1-4 of this paper and in which there exists no arc. The example is a subspace of the Cartesian plane.

Suppose that $n$ is a positive integer, and each of $i$ and $j$ is an integer. Let $K_{n i j}$ denote the square in the plane whose vertices are the points $\left(i / 2^{n}, j / 2^{n}\right)$, $\left([i+1] / 2^{n}, j / 2^{n}\right),\left(i / 2^{n},[j+1] / 2^{n}\right)$, and $\left([i+1] / 2^{n},[j+1] / 2^{n}\right)$. Let $A_{n i j}$ be a pseudoarc (for a description of a pseudoarc, see [1]) which contains the two points $\left(i / 2^{n}, j / 2^{n}\right)$ and $\left([i+1] / 2^{n},[j+1] / 2^{n}\right)$ and, except for these points, lies in the interior of $K_{n i j}$, and let $B_{n i j}$ be a pseudoarc which contains the two points $\left(i / 2^{n},[j+1] / 2^{n}\right)$ and $\left([i+1] / 2^{n}, j / 2^{n}\right)$ and, except for these points, lies in the interior of $K_{n i j}$. Then let $S$ denote $\bigcup\left\{A_{n i j} \cup B_{n i j}: n\right.$ is a positive integer and each of $i$ and $j$ is an integer $\}$. It is easily seen that the space $S$ satisfies Axioms 1-4 of this paper, and it is of some interest to note that $S$ has dimension 1.

THEOREM 4.1. $S$ contains no arc.

Proof. Suppose that $S$ contains an arc $A . S$ is the union of countably many pseudoarcs $S_{1}, S_{2}, \cdots$. If $n$ is a positive integer and $A$ and $S_{n}$ intersect, then $A \cap S_{n}$ is closed and totally disconnected, for $S_{n}$ contains no arc. Then the compact continuum $A$ is the union of countably many closed totally disconnected sets. But this is contradictory [2, Chapter I, Theorem $\left.44^{\prime}\right]$. Hence $S$ contains no arc.

\section{REFERENCES}

1. R. H. Bing, A homogeneous indecomposable plane continuum, Duke Math. J. vol. 15 (1948) pp. 729-742.

2. R. L. Moore, Foundations of point-set theory, Amer. Math. Soc. Colloquium Publications, vol. 13, New York, 1932.

State University of Iowa, Iowa City, Iowa 